\title{
PARES EVALUADORES: UNA CONSTANTE TRIBULACIÓN PARA LOS EDITORES DE REVISTAS INDEXADAS
}

\section{PEER REVIEW: A PERMANENT TRIBULATION FOR EDITORS OF INDEXED JOURNALS}

\author{
Ingeborg Zenner de Polanía ${ }^{1}$ \\ ${ }^{1}$ I.A., Ph.D. Universidad de Ciencias Aplicadas y Ambientales, U.D.C.A, calle 222 No. 55-37. Bogotá. D.C.-Colombia.
}

Rev. U.D.C.A Act. \& Div. Cient. 17(2): 597-605, Julio-Diciembre, 2014

\section{RESUMEN}

La mayoría de las publicaciones seriadas, colombianas y suramericanas, no cobran por página impresa, lo que dificulta lograr la evaluación por árbitros externos, cualificados y desinteresados. Esta reflexión analiza la búsqueda de evaluadores, los problemas que surgen, las negativas de los pares, los incumplimientos, pero también las colaboraciones positivas de profesionales nacionales e internacionales. Abarcando dos años y medio de análisis, se muestra que, en promedio, el 54,88\% de los posibles pares responde y, de ellos, se compromete un $77,51 \%$. Un recorderis enviado a un $31,19 \%$ no surtió efecto, únicamente un 13,17\%. La mayoría de los pares solamente diligencia el instrumento de evaluación; hasta un 53,33\% realiza una compleja y completa revisión y conceptualización. Los académicos del exterior son colaboradores positivos, destacándose los brasileros. Sobresalen entre los colombianos, los profesores de la Universidad Nacional de Colombia, de la Universidad del Valle, de la Universidad de Antioquia y de la Universidad de Córdoba.

Palabras clave: Evaluación manuscritos, publicaciones seriadas, revisión, cumplimiento.

\section{SUMMARY}

Most Colombian and South-American indexed journals do not charge fees for printed pages, having as consequence difficulties to achieve the collaboration of expert and disinterested peer reviewers. In this study the search for reviewers, the problems which arise, the denial of the peers, the non-fulfillment, but also the positive collaboration of national and international professionals is analyzed. Including two and a half years of revision it is shown that, an average of $54.88 \%$ of the possible reviewers responds and of them a $77.51 \%$ is committed. A recorderis sent to a $31.19 \%$ was without response in a $13.17 \%$ of the compromised academics. Most peers only fill out the evaluation form; however up to a $53.33 \%$ reali- zes a complex and complete evaluation. Nonnative reviewers are positive collaborators, standing out Brazilian researchers. Between Colombian peers those of the Colombian National University, the "Universidad del Valle", the "Universidad de Antioquia" and the "Universidad de Córdoba" can be distinguished.

Key words: Manuscript evaluation, serial publications, review, fulfillment.

\section{INTRODUCCIÓN}

Una de las preguntas más frecuentes de los autores antes de someter un manuscrito a una revista es ċcuánto se demoran los trámites? La respuesta siempre comienza con la palabra "depende", lo que se puede deducir de la figura 1, en la que se indican los pasos básicos que deben seguir los manuscritos, desde su sometimiento hasta el envío a la imprenta. Depende de la calidad del texto, de la agilidad de los autores de responder a las inquietudes que arroja una primera revisión interna y, en un alto porcentaje, del cumplimiento de los pares evaluadores. Obedece, ante todo, de lograr el consentimiento de profesionales idóneos, que ejercen la función de árbitro externo y de la atención de ellos en actuar como colaboradores desinteresados, ya que solamente unas pocas revistas se dan el lujo de poder retribuir económicamente la labor de los evaluadores.

De acuerdo a lo expresado por Campanario (2002), el "sistema de revisión por expertos" radica en que dos o más evaluadores revisan y analizan el contenido de los manuscritos, para "determinar tanto la validez de las ideas y los resultados como su impacto potencial en el mundo de la Ciencia." Los evaluadores deben, por lo general, ser académicos de trayectoria investigativa reconocida, con postgrado, conocedores del tema y autores de artículos publicados recientemente en revistas indexadas. La revisión por pares o "peer review", 


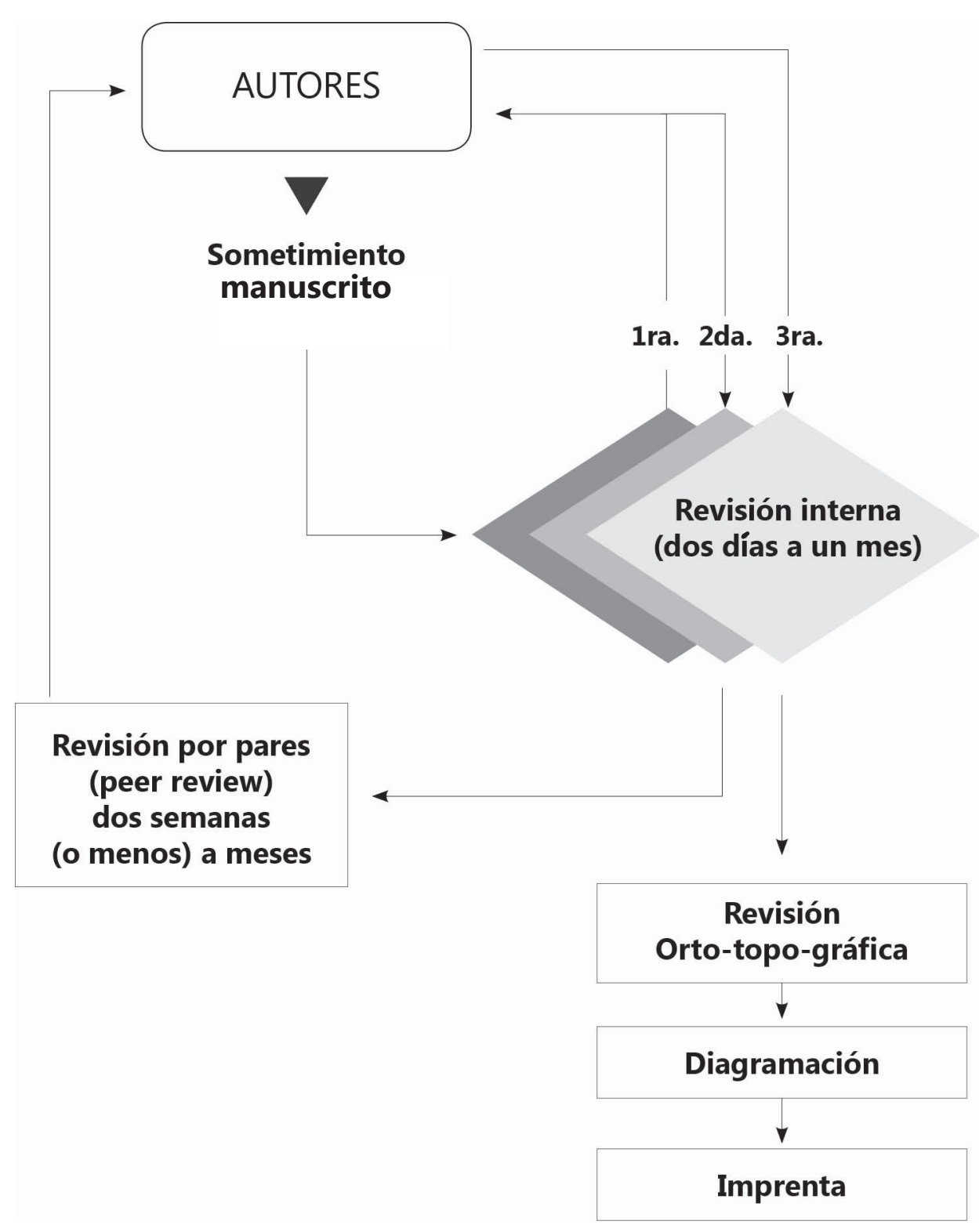

Figura 1. Pasos que debe seguir un manuscrito desde el sometimiento hasta la impresión del artículo.

de acuerdo a la Lloyd Sealy Library (2013), corresponde al proceso de lograr que expertos en la temática tratada lean y comenten resultados de investigación, para validar y certificar su calidad; el propósito es asegurar la calidad de textos sometidos a la revista. Se debe determinar si el texto sometido es original, no editado previamente y si es de calidad y pertinente (Martínez, 2012). Los académicos colaboradores revisan la precisión y valoran la validez de la metodología empleada y prestan especial cuidado a la interpretación y discusión de los resultados. Siendo conocedores del tema tratado tienen la capacidad de detectar plagio, uno de los problemas a los que se ve enfrentado el editor. Pueden rechazar el escrito, si consideran que carece de validez y de rigor científico, pero ante todo deben realizar una labor ética y colaboradora (Hames, 2013).

Aunque la revisión por pares ha sido criticada por no cumplir necesariamente con el aseguramiento de la calidad de un manuscrito, también es cierto que una revista que no cuenta con el sistema de "peer review" no es considerada seria, por 
lo que las publicaciones seriadas resaltan y requieren este aspecto (Nielsen, 2009). Las revistas en sus normas deben declarar, por ejemplo, sus políticas respecto al sistema de revisión por expertos que emplean, aunque existen pocos instructivos para los autores que aclaran concretamente este procedimiento (Tavares de Matos Cardoso, 2011). Además, todas las instituciones indexadoras, como ISI, Scopus, SciELO, entre otras, dan un alto valor a esta particular exigencia (Martínez, 2012).

Por lo general, las revistas usan el sistema de "doble ciego", es decir, ocultar la identidad de autores y de evaluadores; ni los autores ni los evaluadores se conocen entre sí. Este método es prácticamente universal, a pesar que existen estudios que demuestran que los resultados proporcionados por desconocimiento o conocimiento o una combinación entre los dos sistemas, de autores y referees, no afecta la cualidad de las revisiones, de manera estadísticamente significativa (Van Rooyen et al. 1999).

La gran mayoría de las publicaciones seriadas, nacionales e internacionales, cubren algún tema perteneciente a un programa o área de la ciencia. En este caso, se facilita la búsqueda de árbitros, por ejemplo, en la base de datos de pares de Scienti Colciencias (Departamento Administrativo de Ciencia, Tecnología e Innovación), Colombia. Para revistas multidisciplinarias que abarcan varios programas, la búsqueda y el hallazgo de árbitros competentes es mucho más complejo y demorado.

Una vez escogido el experto, la evaluación lo faculta para definir si un manuscrito puede ser considerado como aceptable "con cambios menores", con "modificaciones mayores" o "rechazado". Depende del autor acatar los cambios sugeridos; hasta tanto el editor considera que las exigencias del par han sido satisfechas (Hirst, s.f.). Resalta la misma autora que problemas inherentes a este procedimiento son, en primera instancia, el tiempo entre el sometimiento y la publicación y, en segundo término, la dificultad de obtener evaluadores que disponen del tiempo y de la inclinación para proporcionar revisiones pensadas y constructivas.

Entre los criterios generales de clasificación para fines de indexación de una publicación seriada establecidos por Colciencias (2013) figuran, en primera instancia, la "Calidad Científica", la cual, entre otros requisitos, exige que el documento haya sido "objeto de evaluación por pares". Para acceder una revista a determinada categoría, los expertos evaluadores deben cumplir con ciertas características, establecidas por la guía de Colciencias, dentro de las "Condiciones para la clasificación de revistas en las categorías del Índice Bibliográfico Nacional Publindex -IBN Publindex". Para estar categorizada en $\mathrm{B}$, el $60 \%$ de los árbitros deben pertenecer a instituciones diferentes a aquella que edita la revista y el 30\% de ellos, con formación en maestría. Además, haber publicado al menos un artículo de investigación e innovación, en los dos años anteriores al período de la indexación, en una revista diferente a aquella en la que colaboran como evaluador de manuscritos. Para la categoría A2 y A1, rigen las mismas exigencias, adicionado con que el $20 \%$ de los árbitros tienen que ser de instituciones extranjeras. Este último aspecto puede ser considerado positivo, pues como lo menciona Harrison (2004), la escogencia de pares evaluadores procedentes de diferentes países y con variadas perspectivas, puede evitar prácticas inadecuadas y manipuladas.

Evans et al. (1993) establecieron las características que debería poseer un par evaluador competente y estudiaron 226 referees de 131 manuscritos. Detectaron que si un par era menor de 40 años, pertenecía a una institución de educación superior acreditada y desconocía la identidad de los autores, existía una probabilidad del $87 \%$ que iba a proporcionar una buena revisión, mientras que evaluadores sin ninguna de estas características, solamente tenían un 7\% de posibilidad de responder positivamente. Otras características mencionadas, significativas sólo en un análisis bivariado, fueron entrenamiento en investigación y títulos de postgrados.

Los objetivos de la presente reflexión fueron el análisis de la búsqueda, por parte de un editor de una revista multidisciplinaria, de pares evaluadores competentes, los problemas que surgen, las negativas de los académicos, los incumplimientos, pero también las colaboraciones positivas y relevantes de profesionales nacionales e internacionales.

\section{MATERIALES Y MÉTODOS}

Para esta investigación, se escogieron y analizaron los trámites realizados para lograr la revisión por pares evaluadores de los manuscritos sometidos y, actualmente, ya publicados en la Revista U.D.C.A Actualidad \& Divulgación Científica, publicación oficial multidisciplinaria de la Universidad de Ciencias Aplicadas y Ambientales U.D.C.A, Bogotá, Colombia. Se examinaron los antecedentes del arbitraje de los manuscritos sometidos y aceptados en el segundo semestre de 2011 y en los años 2012 y 2013, es decir, para un total de cinco fascículos. Se tabularon los datos de las solicitudes a árbitros que cumplían con los requisitos de Publindex, sus respuestas y su cumplimiento en la evaluación de los manuscritos.

La selección inicial de los evaluadores, se realizó basada en su hoja de vida, revisando, entre otros aspectos, sus características académicas y sus publicaciones. Como soporte inicial, se tuvo la oferta de pares en Scienti Colciencias, los autores de las publicaciones registradas en Scielo org de los países donde existe esta base de datos, con énfasis en Colombia y las hojas de vida de los candidatos (CvLac, Lattes); ocasionalmente, se recurrió a Google Académico y cuando 
el texto recibido era en idioma inglés, a autores con publicaciones en revistas en esta lengua, homologadas.

Para fines de discusión, se revisó el CvLac del $\pm 50 \%$ de los pares inscritos en Scienti Colciencias, a principios de enero de 2014, escogiendo para esta labor los programas de Medicina, Agronomía, Matemáticas y Zoología.

Al solicitar la colaboración a los posibles árbitros, se anexa, para su información, el resumen del manuscrito a evaluar y se indica que la revisión corresponde a lo denominado "doble ciego". Al aceptar el académico, recibe el texto y el instrumento de evaluación, en el que debe consignar su concepto. Sobre el texto, se sugiere que el árbitro realice comentarios, correcciones y sugerencias, con el objetivo de completar y mejor el escrito.

Algunos árbitros, en especial los investigadores brasileros, registran en su hoja de vida su actividad como árbitro; por tanto, en cada fascículo de la revista U.D.C.A, se publican sus nombres y su afiliación institucional Estos datos generales publicados, se tabularon por país de procedencia, nacionales e internacionales, de los colaboradores evaluadores.

Los resultados obtenidos, se analizaron con un alcance descriptivo-comprensivo, con lo que se pretendió lograr un acercamiento más sensible y abierto hacia los procesos y las actuaciones de los árbitros externos, de manuscritos sometidos a la revista.

\section{RESULTADOS Y DISCUSIÓN}

La Revista U.D.C.A Actualidad \& Divulgación Científica, publicación seriada multidisciplinaria cubre, principalmente, artículos dentro de algunos de los programas especificados por Colciencias: Ciencias de la Salud (Medicina, Enfermería, Educación Física), Ciencias Agrarias (con énfasis en Agronomía, Ciencia y Tecnología de Alimentos, Medicina Veterinaria y Zootecnia), Ciencias Exactas y de la Tierra (Física, Matemáticas, Química) y, ocasionalmente, Ciencias Biológicas y Ciencias Humanas.

Igual a como lo menciona Campanario (2002), la selección de los revisores de manuscrito corresponde al editor de la revista. Se asume que la persona posee el conocimiento para seleccionar los expertos apropiados para determinado manuscrito sometido, perteneciente a una disciplina específica. Esto no lo puede cumplir un editor de una revista multidisciplinaria, pues sus conocimientos se limitan necesariamente a determinada área o programa.

En el presente caso, los autores de los escritos sometidos tienen la posibilidad de sugerir árbitros externos. Si ellos sugieren pares, la hoja de vida es analizada, no solamente por el cumplimiento de los estándares de la revista, sino también desde el punto de vista de posibles conflictos de intereses o endogamia, tales como pertenecer al mismo programa de determinada universidad o haber publicado artículos conjuntamente con los autores del texto actual; sin embargo, como soporte básico para contactar a posibles revisores, se consulta la oferta de pares en Scienti, ya que para los evaluadores inscritos en cada una de las áreas de conocimientos, Colciencias ofrece un sistema amigable de búsqueda, que permite acceder, directamente, una vez conocido el área en el que el científico se inscribió y relacionado con el manuscrito sometido, a la hoja de vida del profesional.

Infortunadamente, muchos de los posibles pares evaluadores inscritos no cumplen con el requisito de Publindex y que rige para la indexación de una revista, de haber publicado, en los dos años anteriores a la indexación, al menos un artículo de investigación e innovación, en una revista diferente a aquella en la que colaboran como evaluador. En enero de 2014, asumiendo la actualización del CvLac de los pares, la confrontación de la oferta de pares en Scienti con esta exigencia, revisando un programa de cada uno de las áreas principales mencionadas, tuvo los siguientes resultados: en Ciencias de la Salud, donde se había escogido Medicina, se detectó la inscripción de 285 pares evaluadores; al azar se revisó la hoja de vida de 143 , encontrándose que el $27,97 \%$ no mostró artículo publicado durante 2012 y 2013. Llamó la atención que muchos de los pares registrados en esta área mencionaron, dentro de su producción bibliográfica, resúmenes presentados en eventos, lo que no es aceptable como un artículo. Publindex admite tres categorías para este fin: artículos científicos, artículos de revisión y artículos de reflexión. En este caso particular, se justifica la pregunta: ¿Por qué los médicos no publican los hallazgos presentados en Congresos?

En el área de Agronomía, se evidenció un total de 181 pares inscritos y se revisó el CvLac de 91 de ellos, con el resultado que el $42,85 \%$ no cumplió con el requisito mencionado, mientras que más del $50 \%$ sí actuó como autor principal o coautor de artículos de cualquiera de las tres tipologías aceptadas. Matemáticas fue el área tomada dentro del programa de Ciencias Exactas y de la Tierra; de los 82 pares inscritos, se exploró el currículo de 41 profesionales, lo que mostró una ausencia del cumplimiento de la exigencia del 41,46\%. Finalmente, se examinó en Ciencias Biológicas, el área de Zoología, con 84 evaluadores inscritos, de quienes se examinó el CvLac del 50\%, hallándose que el 35,71\% de los profesionales que se registraron como pares no habían realizado publicaciones de artículos de cualquiera de las tres categorías aceptadas, en revistas indexadas.

Estos resultados reflejan que, aparentemente, los investigadores colombianos se acogieron a la "Convocatoria nacional para el reconocimiento y medición de grupos de investiga- 
ción, desarrollo tecnológico o innovación y para el reconocimiento de investigadores del sistema nacional de ciencia, tecnología e innovación 2013" de Colciencias y actualizaron sus hojas de vida. Esta deducción, se basa en que en años anteriores se observaba que los porcentajes de profesionales que cumplían con el requisito de haber publicado por lo menos un artículo de la tipología en los dos años anteriores eran menores, lo que dificultaba la detección de pares evaluadores, para los manuscritos sometidos a la revista (datos no presentados).

Para muchos profesionales, ante todo jóvenes que están iniciando su carrera de investigadores y que ya han logrado un postgrado, ser contactado, como lo expresan en sus respuestas, para colaborar como "referee" o experto, es un honor. Son ellos que, por lo general responden, lo que a menudo no lo hacen los investigadores de conocida trayectoria, pues sus múltiples ocupaciones no se lo permiten. Campanario (2002) menciona el mismo aspecto, reforzado por el estudio de Stossel (1985), quien encontró que a menudo se observa una baja calidad de evaluación de los pares de alta gama en comparación con "las revisiones calificadas de alta calidad, "ejecutadas por "referees de menor estatus".

En la actualidad, se puede ver que, en general, en las áreas revisadas, se destacan las publicaciones de los médicos, seguido por los investigadores que se dedican a la Zoología. Porcentajes muy similares, 42,85 y $41,46 \%$, respectivamente, de ausencia de productos, resultados de investigaciones, los muestran los profesionales de la agronomía y los matemáticos. ¿Será que esto se debe a la falta de financiación apropiada de investigaciones pertinentes o a la ausencia de inclinación hacia la ciencia, de los primeros y la dificultad de encontrar temática apropiada, de los segundos?

Después de Scienti Colciencias sigue en relevancia Scielo, especialmente Scielo Brasil, en la búsqueda de árbitros externos. Las publicaciones recientes y sus autores se examinan usando, por ejemplo, palabras clave, que conducen a artículos que versan sobre temas similares o relacionados con el manuscrito, para el que se requieren evaluadores.

Comúnmente, una vez detectado un posible par evaluador, se le formula una solicitud de colaboración, que reza: "La revista no cobra por página impresa de artículos publicados, por lo cual, no se dispone de fondos para retribuir el trabajo realizado por los evaluadores". Además, se menciona que como agradecimiento por su invaluable labor como evaluador, recibirá el fascículo de la revista en formato pdf, donde se publica el artículo revisado; igualmente, el número respectivo incluye una página con los nombres e instituciones de los árbitros, acompañado de unas líneas de agradecimiento. Se aclara, además, que el tiempo disponible para la revisión del texto es de dos semanas.
Aceptada la evaluación, cada profesional recibe el formato de evaluación y se le indica que "el evaluador es anónimo y tiene la oportunidad de realizar observaciones y correcciones al texto. Igualmente, sugerencias de cambios, de inclusión de bibliografías adicionales y de otros ajustes para mejorar el escrito, son bien venidas".

En la tabla 1, se muestran los resultados obtenidos y la secuencia de respuestas, una vez contactado un candidato. Se aclara que, aparentemente, algunos números no siguen una secuencia, matemáticamente lógica, lo que se debe a la necesidad ocasional de tener que repetir las solicitudes a otros árbitros o al hecho, que un revisor evaluó más de dos manuscritos.

Para los fascículos revisados, del más reciente al del 2011, el porcentaje de respuestas recibidas fue, respectivamente, del 48,33; 51,63; 55,00; 59,80 y 60,29\%. En el estudio multivariado de Evans et al. (1993), los autores evidenciaron respuestas positivas del $91 \%$ de los pares, quienes tenían características diferentes a las exigidas por Publindex, entre las cuales, se destacaba la edad menor de 40 y la procedencia de una Universidad reconocida. Otra investigación relacionada mostró una respuesta general del $64,7 \%$ de los revisores (Snell \& Spencer, 2005).

No se esperaba que las respuestas positivas por parte de los pares disminuyeran a medida que aumentaba el número de artículos publicados, lo que significaba una cantidad mayor de manuscritos recibidos, consecuencia de una considerable aceptación de la revista, como medio de publicación de los resultados de investigación de los científicos colombianos, es decir, un mayor reconocimiento. Esto, en teoría, debería haber tenido una influencia positiva en la aceptación de la tarea honrosa de ser un "referee". Para el mismo orden de los fascículos, el porcentaje de aceptación fue del 68,96; 78,48; 77,$48 ; 75,40$ y 85,36 ; otras respuestas correspondieron a "falta de tiempo", "no dominio del tema", "próximas salidas de campo" y "viajes al exterior".

El tiempo estipulado para recibir la evaluación corresponde a dos semanas. Muy pocos pares ejecutan la revisión en este tiempo y, a veces, por incumplimiento con lo acordado, se envía un recorderis. Durante el lapso que comprende la investigación, se remitió un recorderis, en promedio, a un $31,19 \%$ de los pares que se habían comprometido; esto surtió efecto positivo en un $86,83 \%$ de ellos; el restante porcentaje, simplemente, no respondió. La ausencia de una respuesta exige iniciar de nuevo el proceso de búsqueda de otro experto, lo que atrasa, considerablemente, la publicación del artículo, en detrimento de las aspiraciones de los autores.

Como se puede detectar de la tabla 1, para cada uno de los 128 artículos que se publicaron en el lapso considerado, se 
Tabla 1. Seguimiento a la búsqueda de pares evaluadores.

\begin{tabular}{|c|c|c|c|c|c|c|c|}
\hline \multirow{2}{*}{$\begin{array}{c}\text { Fascículo } \\
\text { (semestre) } \\
\text { Artículos } \\
\text { publicados }\end{array}$} & $\begin{array}{c}\text { Contac- } \\
\text { tados }\end{array}$ & $\begin{array}{c}\text { Respon- } \\
\text { dieron }\end{array}$ & $\begin{array}{c}\text { Acep- } \\
\text { taron }\end{array}$ & $\begin{array}{c}\text { Cum- } \\
\text { plieron }\end{array}$ & $\begin{array}{c}\text { Recorderis } \\
\text { con } \\
\text { respuesta }\end{array}$ & $\begin{array}{c}\text { Recorderis } \\
\text { sin respuesta }\end{array}$ & $\begin{array}{c}\text { Concepto } \\
\text { más } \\
\text { texto con } \\
\text { comenta- } \\
\text { rios }\end{array}$ \\
\hline $16(2)(2013$ B) 31 & 180 & 87 & 60 & 60 & 29 & 10 & $32 / 60$ \\
\hline $16(1)(2013$ A) 30 & 153 & 79 & 62 & 57 & 21 & 12 & $17 / 57$ \\
\hline $15(2)(2012$ B) 25 & 120 & 66 & 51 & 52 & 14 & 3 & $16 / 52$ \\
\hline $15(1)(2012$ A) 23 & 102 & 61 & 46 & 43 & 9 & 6 & $9 / 43$ \\
\hline $14(2)(2011$ B) 18 & 68 & 41 & 35 & 33 & 8 & 3 & $8 / 33$ \\
\hline
\end{tabular}

contó, finalmente, con la colaboración real de dos evaluadores por manuscrito; a menudo, de tres, si había discrepancia marcada ente los conceptos y, además, de vez en cuando un árbitro colaboraba con la evaluación de más de un texto.

Muchos evaluadores se limitan a llenar el formato de evaluación de manera muy simple, respondiendo a las preguntas con un sí o un no; sin embargo, algunos realizan comentarios, correcciones y propuestas, tanto en el instrumento como en el manuscrito; efectúan una evaluación completa y compleja, que permite a los autores complementar y perfeccionar el escrito y al editor tomar una decisión de publicar o no el texto sometido, una vez formalizados los ajustes por los autores (Tabla 1). Promediando los datos de la última columna de esta tabla, se obtiene que el 53,33\% de los pares evaluadores efectúa la labor completa. El estudio de Snell \& Spencer (2005), encontró que un $87 \%$ de los revisores complementaron y devolvieron el texto con comentarios, fuera de haber diligenciado el formato de evaluación. Ojalá en un futuro se logre un acercamiento a este porcentaje, significativamente mayor, que el obtenido en este estudio.

En la tabla 2, se consigna el título académico y la procedencia de los pares evaluadores colaboradores, en el lapso que comprende este estudio. Entre los nacionales, casi siempre se distinguen los académicos de la Universidad Nacional de Colombia (U.N.), aunque entre ellos se registran también a los profesionales que no responden a las solicitudes de arbitraje (datos sin incluir). De esta deducción, no se puede concluir que los docentes de la U.N. son simultáneamente los más o los menos juiciosos y colaborativos; se considera que el resultado final satisfactorio, se debe al mayor número de profesionales que cumplen con los requisitos y que, además, laboran en la universidad más grande del país, que ofrece el mayor número de programas académicos. Entre las otras universidades que se destacan, se debe tener en cuenta que solamente se mencionan en la tabla las primeras cuatro, pues no se detectó una marcada tendencia de colaboración.
Se puede deducir que académicos de la Universidad de Antioquia, del Valle, de Córdoba y de la UPTC, siempre han favorecido este proceso, de manera desinteresada. No se debe olvidar que una revista multidisciplinaria, como la analizada, siempre va a requerir un número elevado de pares, expertos de las más diversas disciplinas y áreas del conocimiento, por lo que no se puede concluir que los profesionales de una $u$ otra institución de educación superior son mejores colaboradores desinteresados, que aquellos de otra universidad.

Esta última afirmación es especialmente aplicable al apoyo de académicos de otros países y universidades; no obstante, siempre sobresale la eficiencia de colaboración de profesionales, docentes-investigadores de Brasil (Tabla 2). Ellos, además incluyen en su hoja de vida, la vinculación como árbitros a determinadas revistas. Entre el sinnúmero de universidades que existen en Brasil y cuyos docentes han colaborado, no fue posible hallar una tendencia. Dependiendo del tema del manuscrito, con la ayuda de las palabras clave, se busca el evaluador basado en su profesión, su título y sus publicaciones, sin tener en cuenta la universidad a la que pertenece. Son también los brasileros que, al considerar que no son expertos en el tema, sugieren otros nombres. Además, tienen la ventaja aparente sobre los académicos de otros países que registran una alta productividad científica, que facilita su escogencia como par evaluador. Otros países latinoamericanos que proporcionan árbitros competentes son Argentina, Chile y Venezuela; no se quedan atrás Cuba y México. Entre los académicos europeos resalta, lógicamente, por el dominio del idioma, España.

De la misma tabla 2, se deduce que la gran mayoría de los pares poseen el título de doctor y todos tienen, como mínimo, una maestría, como lo exige Publindex. La revista U.D.C.A busca que sus colaboradores evaluadores ostentan el doctorado, pues se considera que, dada su experiencia investigativa y su producción intelectual, sus aportes validan $y$, hasta cierto punto, certifican la calidad científica de los 
Tabla 2. Título académico y procedencia de los pares colaboradores.

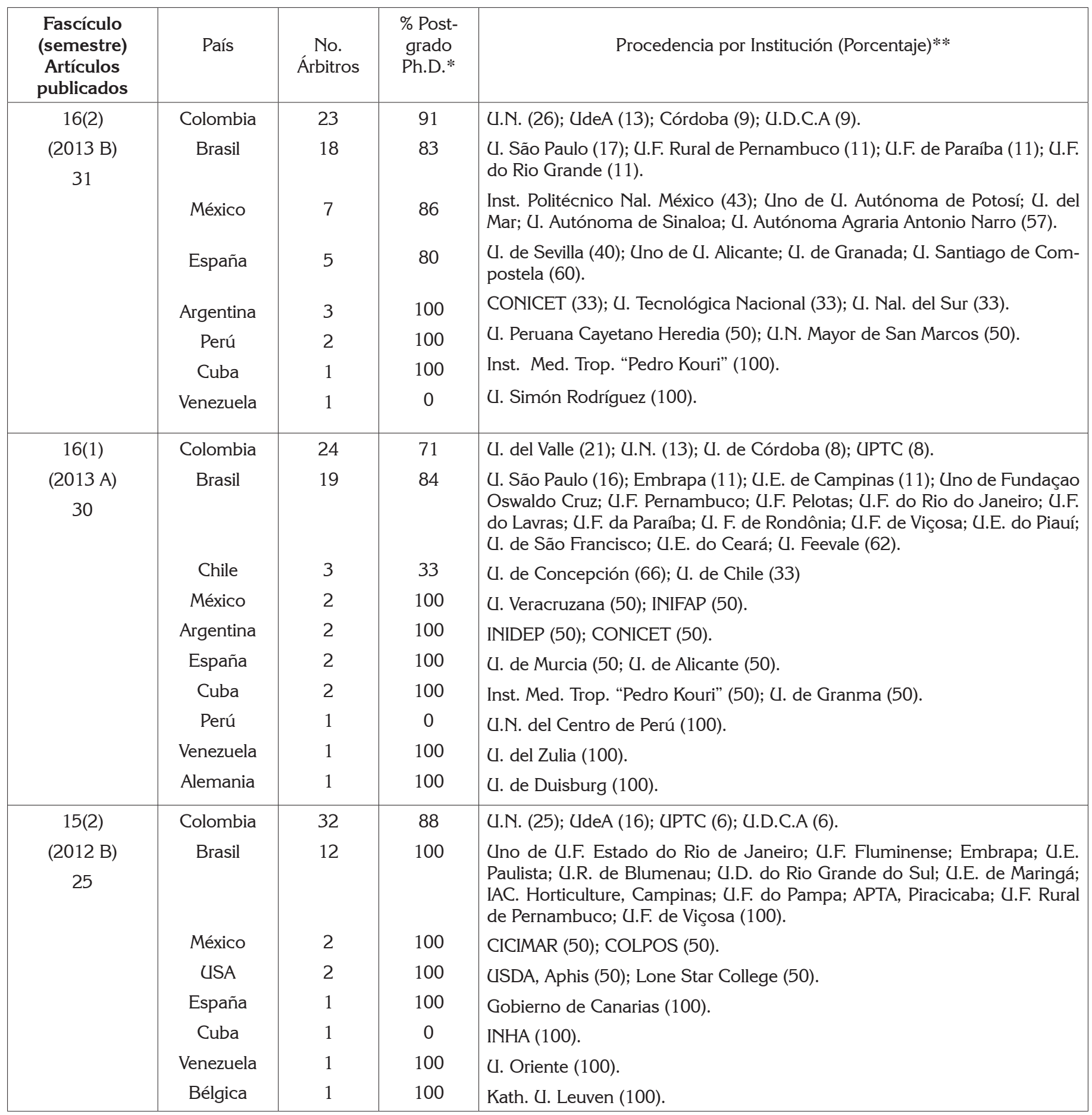


Continuación tabla 2.

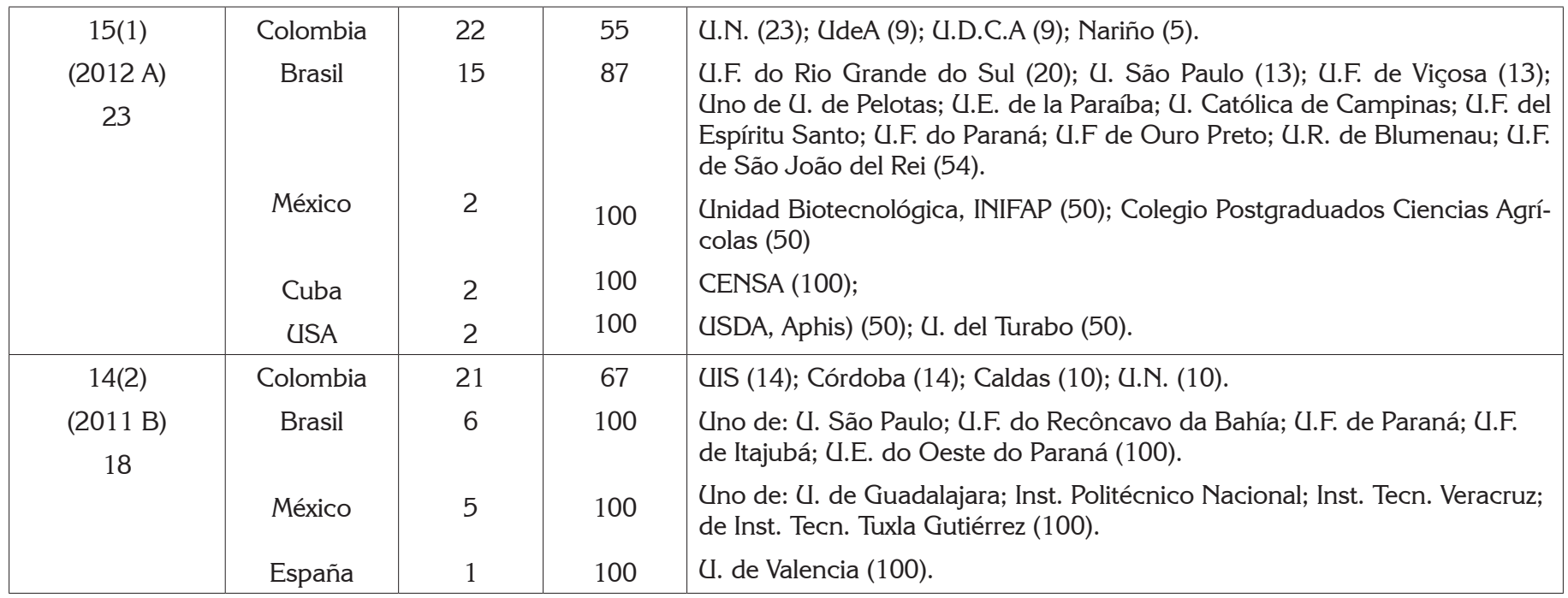

* Los porcentajes restantes corresponden a maestrías.

** Sólo se mencionan las primeras cuatro universidades o instituciones.

textos. Además, sus contribuciones logran una mejoría de un manuscrito, para poderlo publicar o proporcionan las bases científicas para no aceptar un escrito, es decir, colaboran eficientemente, con el trabajo de los editores.

Aunque en el parágrafo anterior se indican aspectos positivos de la colaboración de un par, siempre cabe la pregunta concreta, como la formula Campanario (2002): "¿Qué ganan, pues, al colaborar con las revistas?" Fuera de recibir un reconocimiento extraoficial y oficial de un editor de una revista, el árbitro externo amplía su horizonte y recibe, de pronto, inspiraciones para formular sus propias futuras investigaciones; una vez publicado el artículo, se puede comunicar con los autores para, de pronto, formar redes o complementar los grupos de investigación. Además, se supone valioso en esta reflexión general copiar fielmente la frase escrita por Campanario (2002): ".....no cabe duda de que muchos investigadores consideran que la revisión de los trabajos es uno de los deberes básicos de la comunidad científica, siempre con el fin de contribuir a aumentar el rigor y la validez al conocimiento que se genera", con la cual, estamos muy de acuerdo. Con una revisión completa y compleja, con críticas constructivas y con comentarios productivos, podemos ayudar a formar investigadores jóvenes, pensantes y comprometidos con la Ciencia y la Tecnología.

Finalmente, uno de los puntos críticos es representado por el tiempo (datos no presentados) que requieren los evaluadores para la revisión, a pesar de comprometerse con un período de dos semanas. Muchas veces, se toman hasta meses, no justificados, con algún imprevisto. Aún peor es la actitud de algunos, afortunadamente de pocos, que aceptan y, luego, a pesar de comunicaciones, simplemente nunca responden.
A ellos se les olvida que los autores esperan una respuesta experta, así sea negativa, dado que con estos análisis, el autor tiene bases para introducir cambios, aumentar la calidad y revisar literatura adicional, para poder lograr la publicación del artículo.

El lapso de dos semanas para la revisión detallada y reflexiva de un manuscrito, que por lo general no abarca más de 25 páginas a doble espacio, se considera más que suficiente; inclusive, un revisor puede evaluar un escrito en una lapso mucho menor, como lo indican las observaciones de McNutt et al. (1990). Estos autores y también Snell \& Spencer (2005), mencionan que el tiempo promedio de dedicación juiciosa a la evaluación de un texto corresponde a tres horas, aunque existen manuscritos que demandan seis o más horas, pues deben ser revisados dos o más veces. Los pares evaluadores deben, por lo tanto, antes de comprometerse, analizar sus agendas y determinar si disponen o no del tiempo requerido, para luego no incurrir al incumplimiento.

Uno de los puntos críticos para lograr una respuesta positiva y cumplimiento parece ser la ausencia de motivación para colaborar como evaluador. ¿̇Será que este punto mejorará, ahora que los revisores pueden incluir en su CvLac la actividad como par evaluador?, ¿̇será que los y las colaboradoras preferirían, como los pares brasileros, no ser anónimos? De todos modos, a corto plazo, con muy pocas excepciones, los evaluadores externos no podrán contar con un estímulo monetario, pero sí con los decididos agradecimientos de los editores y de los autores.

De los resultados presentados, se puede concluir que una de las labores menos gratificantes de un editor de una re- 
vista indexada es la búsqueda de evaluadores competentes y cumplidores. Formular recorderis, esperar respuestas que no llegan, iniciar una nueva revisión de posibles académicos que puedan colaborar, requiere tiempo adicional, que un editor podría emplear en muchas otras actividades, más interesantes y productivas, en beneficio de la publicación. Por el otro lado, las respuestas positivas, las sugerencias y los comentarios de los pares, entre otros aspectos, aportan enormemente en ampliar el horizonte académico de un editor y lo educan para cumplir su tarea, de una manera más eficiente.

Agradecimientos: A todos los pares que de una u otra manera han colaborado para presentar al lector de la Revista U.D.C.A Actualidad \& Divulgación Científica artículos de calidad, que han permitido su categorización en A2. Conflicto de Interés: La autora declara que no existe conflicto de intereses que ponga en riesgo la validez de los resultados.

\section{BIBLIOGRAFÍA}

1. CAMPANARIO, J.M. 2002. El sistema de revisión por expertos (peer review): muchos problemas y pocas soluciones. Rev. Esp. Doc. Cient. 25(3):267-285.

2. COLCIENCIAS. 2013. Indexación de Revistas Seriadas de Ciencia, Tecnología e Innovación, Colombianas. Guía Servicio Indexación. 15p. Disponible desde Internet en: http:/www.colciencias.gov.co/sites/default/files/ckeditor_files/files/Gu\%C3\%ADa\%20Servi-

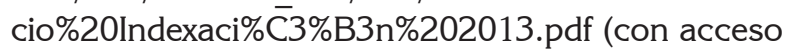
08/10/2013).

3. EVANS, A.T.; McNUTT, R.A.; FLETCHER, S.W.; FLETCHER, R.H. 1993. The characteristics of peer reviewers who produce good-quality reviews. J. Gen. Int. Medicine. 8(8):422-428.

4. HAMES, I. 2013. COPE Ethical Guidelines for Peer Reviewers. Disponbile desde Internet en: http://publicationethics.org/files/Ethical_guidelines_for_peer_reviewers_0.pdf (con acceso 15/08/2013).

5. HARRISON, C. 2004. Peer review, politics and pluralism. Environm Science \& Policy. 7:357-368.
6. HIRST, K.K. s.f. The Way Peer Review Works in the Social Sciences. Disponible desde Internet en: http:// archaeology.about.com/od/pethroughpg/a/peer_review.htm (con acceso 06/12/13).

7. LLOYD SEALY LIBRARY. 2013. Evaluating Information Sources. What Is A Peer-Reviewed Article? Disponible desde Internet en: http://guides.lib.jjay.cuny.edu/ content.php?pid $=209679 \&$ sid $=1746812$ (con acceso 28/11/2013).

8. MARTÍNEZ, G.S. 2012. La revisión por pares y la selección de artículos para publicación. Rev. Col. Psicol. 21(1):27-35.

9. McNUTT, R.A.; EVANS, A.T.; FLETCHER, R.H.; FLETCHER, S.W. 1990. The effects of blinding on the quality of peer review. J. Am. Med. Assoc. 263:13711374.

10. NIELSEN, M. 2009. Three myths about scientific peer review. Disponible desde Internet en: http://michaeInielsen.org/blog/three-myths-about-scientific-peerreview/ (con acceso 09/12/13).

11. SNELL, L.; SPENCER, J. 2005. Reviewers' perceptions of the peer review process for a medical education journal. Med. Educ. 39(1):90-97.

12. STOSSEL, T.P. 1985. Reviewer status and review quality: Experience of the Journal of Clinical Investigation. New England J. Medicine. 312:658-659.

13. TAVARES DE MATOS CARDOSO, M.M. 2011. El peer review de las revistas científicas en Humanidades y Ciencias Sociales: políticas y prácticas editoriales declaradas. Rev. Esp. Doc. Cient. 34(2):141-164.

14. VAN ROOYEN, S.; GODLEE, F.; EVANS, S.; SMITH, R.; BLACK, N. 1999. Effect of blinding and unmasking on the quality of peer review. J. Gen. Int. Med. 14(10):622-624.

Recibido: Junio 13 de 2014

Aceptado: Septiembre 24 de 2014

Como citar:

Zenner de Polanía, I. 2014. Pares evaluadores: una constante tribulación para los editores de revistas indexadas. Rev. U.D.C.A Act. \& Div. Cient. 17(1): 597-605. 